\title{
Electroretinographic Assessment in Joubert Syndrome: A Suggested Objective Method to Evaluate the Effectiveness of Future Targeted Treatment
}

\author{
Giulio Ruberto - Vincenzo Parisi - Chiara Bertone - Sabrina Signorini • \\ Mauro Antonini · Enza Maria Valente · Federica Manzoni • \\ Valentina Serpieri · Riccardo Fausto · Luciano Quaranta
}

Received: June 3, 2020 / Published online: July 15, 2020

(C) The Author(s) 2020

\section{ABSTRACT}

Introduction: Joubert syndrome (JS) is an autosomal recessive disorder characterized by a congenital malformation of the mid-hindbrain and a large spectrum of clinical features including congenital retinal dystrophy. The function of different retinal elements (rod,

Digital Features To view digital features for this article go to https://doi.org/10.6084/m9.figshare.12582653.

G. Ruberto - C. Bertone - R. Fausto - L. Quaranta Department of Surgical and Clinical, Diagnostic and Pediatric Sciences, Section of Ophthalmology, IRCCS Fondazione Policlinico San Matteo, University of Pavia, Pavia, Italy

V. Parisi ( $\square)$

IRCCS Fondazione Bietti, Rome, Italy

e-mail: vincenzo.parisi@fondazionebietti.it

S. Signorini · M. Antonini

Child Neurology and Psychiatry Unit, IRCCS

Mondino Foundation, Pavia, Italy

E. M. Valente $\cdot$ V. Serpieri

Department of Molecular Medicine, University of Pavia, Pavia, Italy

E. M. Valente

Department of Molecular Medicine, IRCCS

Mondino Foundation, University of Pavia, Pavia, Italy

F. Manzoni

Scientific Direction Clinical Epidemiology and Biometry Unit, San Matteo Hospital Foundation, Pavia, Italy cone, bipolar cells) can be objectively evaluated by electroretinogram (ERG) recordings. Our work aims to evaluate the retinal function (by ERG recordings) in patients with JS with or without congenital retinal dystrophy. In addition, since clinical trials should be performed in the near future in JS, our results could provide information about the possible usefulness of ERG recordings in the assessment of the efficacy of treatments targeted to improve the retinal involvement.

Methods: In this observational and prospective study, 24 children with genetic identification for JS (mean age $10.75 \pm 6.59$ years) and 25 healthy age-similar normal control subjects (control group, mean age $10.55 \pm 3.76$ years) were enrolled. On the basis of the presence/absence of retinal dystrophy at fundus examination, patients with JS were divided into two groups: patients with JS with retinal dystrophy (16 children, mean age $11.00 \pm 6.74$ years, providing 16 eyes; JS-RD group) and patients with JS without retinal dystrophy (8 children, mean age $10.50 \pm 6.45$ years, providing 8 eyes; JS-NRD group). In patients with JS and controls, visual acuity (VA), dark-adapted, light-adapted, and 30-Hz flicker ERGs were performed according to International Society for Clinical Electrophysiology of Vision (ISCEV) standard protocols.

Results: When compared to controls, patients in the JS-RD and JS-NRD groups showed significant abnormalities of the values of dark-adapted, light-adapted, and $30-\mathrm{Hz}$ flicker ERG 
parameters. The ERG and VA changes were not significantly correlated.

Conclusions: Our results suggest that a dysfunction of photoreceptors and bipolar cells occurs in patients with JS with or without retinal dystrophy. The retinal impairment can be detected by ERG recordings and this method should be proposed to evaluate the effectiveness of adequate treatment targeted to improve the retinal impairment in patients with JS.

Keywords: Electroretinogram; Joubert syndrome; Ophthalmology; Retinal dystrophy

\section{Key Summary Points}

Why carry out this study?

Joubert syndrome (JS) is an autosomal recessive disorder characterized by congenital malformation of the midhindbrain and a large spectrum of clinical features including congenital retinal dystrophy.

The function of different retinal elements (rod, cone, bipolar cells) can be objectively evaluated by using different types (darkadapted, light-adapted, and $30-\mathrm{Hz}$ flicker) of electroretinogram (ERG) recordings.

This observational and prospective study was carried out to evaluate the retinal function (by ERG recordings) in patients with JS with (JS-RD group) or without (JSNRD group) congenital retinal dystrophy. Our results should provide information about the possible usefulness of ERG recordings in the assessment of the efficacy of treatments targeted to improve the retinal condition.

\section{What was learned from the study?}

When compared to controls, patients in the JS-RD and JS-NRD groups showed significant abnormalities of the values of dark-adapted, light-adapted, and $30-\mathrm{Hz}$ flicker ERG parameters. The ERG and visual acuity changes were not significantly correlated.
In patients with JS with or without retinal dystrophy, a dysfunction of photoreceptors and bipolar cells occurs and this can be detected by ERG recordings. ERG methods should be proposed to evaluate the effectiveness of adequate treatment aimed to improve the retinal impairment of patients with JS.

\section{INTRODUCTION}

Joubert syndrome (JS) is an autosomal recessive disorder characterized by a congenital malformation of the mid-hindbrain and a large spectrum of clinical features; the disease was first described by Marie Joubert [1] who observed four siblings with cognitive impairment, ataxia, episodic tachypnea, eye movement abnormalities, and cerebellar vermis agenesis. At present, the diagnosis is based on the presence of the typical "molar tooth sign" on brain imaging, characterized by cerebellar vermian hypoplasia, thickened and horizontalized superior cerebellar peduncles, and a deepened interpeduncular fossa [2].

The prevalence of JS has been estimated at approximately $1: 100,000$ in the USA [3]. The pathology can occur as an isolated neurological disorder or can be associated with variable involvement of other organs like the liver, kidney, and retina (defined as "Joubert syndrome related-disorders" $[2,3]$ ).

In patients with JS over 30 genes are known $[4,5]$, all encoding proteins of the primary cilium, a subcellular organelle found on the surface of most cellular types, making JS part of the expanding spectrum of "ciliopathies" [6].

The visual system is also involved [7] and, in particular, congenital retinal dystrophy [8-10], jerky eye movements, nystagmus, strabismus [1], oculomotor apraxia [11, 12], coloboma $[13,14]$, and ptosis and extraocular muscle limitation [15] are described in these patients.

In genetic disorders involving the retinal structures, a current and interesting goal is to increase the retinal function by different types of treatment, including gene therapy. At 
present, there are no reported clinical trials on JS aiming to improve the retinal condition.

Since in JS the visual dysfunction is mainly related to the presence of congenital retinal dystrophy [7-9], a particular interest is to assess the function of different retinal elements (rod, cone, bipolar cells) by using an objective approach such as the recordings of different types (dark-adapted, light-adapted, and $30-\mathrm{Hz}$ flicker) of electroretinogram (ERG) responses, by standardized International Society for Clinical Electrophysiology of Vision (ISCEV) protocols [16].

In rare diseases, such as JS, the monitoring of retinal function over time and/or after specific treatments is a debated topic, because it is not easy to find appropriate tests that can evaluate both visual function and retinal function at the same time. Although ERG is a sensitive objective tool for evaluation of retinal function, it is generally neglected in the list of instrumental tests for accurately monitoring potential changes after targeted innovative treatments. Therefore, our aim was to evaluate the functional condition of different retinal elements (by ERG recordings) in patients with JS with or without congenital retinal dystrophy. In addition, since appropriate clinical trials could be performed in the near future for JS, our results might provide new information on the possible usefulness of ERG recordings to evaluate the efficacy of treatments targeted to improve the retinal condition.

\section{METHODS}

\section{Patients}

In this observational and prospective study, 24 children (mean age $10.75 \pm 6.59$ years) with a neuroradiologically proven "molar tooth sign" and with genetic identification for JS and 25 healthy age-similar (mean age $10.55 \pm$ 3.76 years) control subjects were included. Patients with JS were enrolled at the Department of Child Neurology and Child Psychiatry IRCCS C. Mondino, Pavia, and underwent complete ophthalmological evaluation (see below) at the Section of Ophthalmology,
University of Pavia, IRCCS Fondazione Policlinico San Matteo, Pavia. Patients with JS underwent a complete diagnostic workup including a detailed assessment of renal, liver, and heart function, and received genetic counselling. DNA for genetic studies by a next-generation-sequencing-based analysis of 120 ciliary genes, including 27 genes known to cause JS [17], were available for all 24 enrolled patients.

Patients with JS and controls had a complete ophthalmological examination including best corrected visual acuity (BCVA) assessment. Anterior segment evaluation with a slit lamp was performed depending on the patients' cooperation. Cycloplegic refraction (cyclopentolate $1 \%$ ) and dilated fundus examination with indirect ophthalmoscopy were performed on each patient.

On the basis of the presence/absence of retinal dystrophy at fundus examination, patients with JS were divided into two groups:

- Patients with JS with retinal dystrophy: 16 children, providing 16 eyes (JS-RD group)

- Patients with JS without retinal dystrophy: 8 children, providing 8 eyes (JS-NRD group)

Demographic data, identified mutation, observed type of retinal dystrophy, and visual acuity detected in controls and JS-RD and JSNRD groups are reported in Table 1.

All procedures performed in this study were in accordance with the ethical standards of the institutional and/or national research committee and with the 1964 Helsinki declaration and its later amendments or comparable ethical standards. The study was approved by the local institutional review board (Scientific Committee of Section of Ophthalmology, University of Pavia, IRCCS Fondazione Policlinico San Matteo, Pavia, Italy). Informed consent was obtained from all individual participants included in the study.

\section{Visual Acuity Evaluation}

In verbal children, BCVA was assessed by the modified Early Treatment Diabetic Retinopathy Study (ETDRS) charts (Lighthouse, Low vision products, Long Island City, NY, USA) and 
Table 1 Demographic, identified mutation, type of retinal dystrophy, and visual acuity observed in control subjects and in patients with Joubert syndrome with (JS-RD group) or without (JS-NRD group) retinal dystrophy

\begin{tabular}{llll}
\hline & Controls & JS-RD group & JS-NRD group \\
\hline Number & 25 & 16 & 8 \\
Age (years, mean \pm standard deviation) & $10.55 \pm 3.76$ & $11.00 \pm 6.74$ & $10.50 \pm 6.45$ \\
Sex (male/female) & $16 / 9$ & $10 / 6$ & $8 / 0$ \\
Identified mutation & & \\
C5orf42 & 0 & 2 & 4 \\
CC2D2A & 0 & 2 & 4 \\
KIF7 & 0 & 2 & 0 \\
INPP5E & 0 & 4 & 0 \\
TMEM67 & 0 & 4 & 0 \\
AHII & 0 & 2 & 0 \\
Retinal dystrophy & & & 0 \\
Chorioretinal coloboma & 0 & 4 & 0 \\
Retinal pigment (peripheral and/or macula) & 0 & 8 & 0 \\
Thinning of retinal vessels & 0 & 4 & $0.040 \pm 0.001$ \\
Visual acuity (logMAR, mean \pm standard deviation) & $0.046 \pm 0.072$ & $0.621 \pm 0.361$ & \\
\hline
\end{tabular}

expressed in $\log$ MAR values obtained at the distances of $4,2,1$, and $0.5 \mathrm{~m}$. In preverbal and nonverbal children, BCVA was measured with Teller acuity cards (TACs) and the values were converted to logMAR.

\section{Electroretinography Assessment}

Dark-adapted, light-adapted, and $30 \mathrm{~Hz}$ flicker ERGs (flicker ERG) were performed according to ISCEV standard protocols by using skin electrodes [16].

In the analysis of dark-adapted and lightadapted ERGs we considered a- and b-wave implicit time (IT) and a- and b-wave peak-topeak amplitude $(A)$. In the analysis of flicker ERG the peak-to-peak amplitude $(A)$ was considered (Fig. 1).

\section{Statistical Analysis}

The Anderson-Darling and Kolmogorov-Smirnov tests were applied to verify that data were normally distributed. Only one eye was chosen for each patient.

Differences of values of BCVA and of all ERG parameters between controls and both JS groups were evaluated by the one-way analysis of variance (ANOVA). Pearson's test was used to assess the relationship between the values of BCVA and those of all ERG parameters. All statistical analyses were performed using SPSS version 26 (Statistical Package for Social Science IBM) and a $p$ value less than 0.01 was considered as statistically significant. 
C\#12 eye

D-A ERG

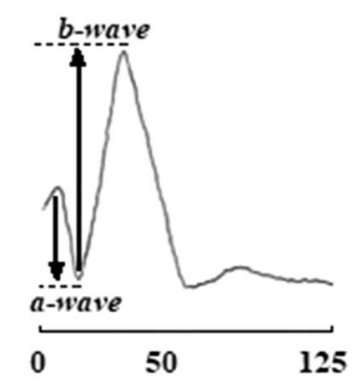

L-A ERG

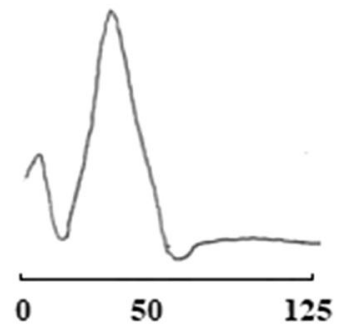

F-ERG

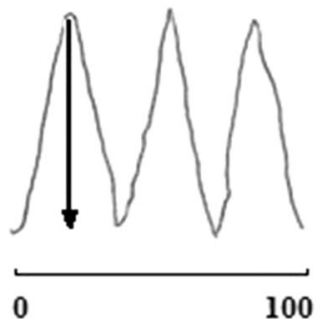

JS-RD\#9 eye
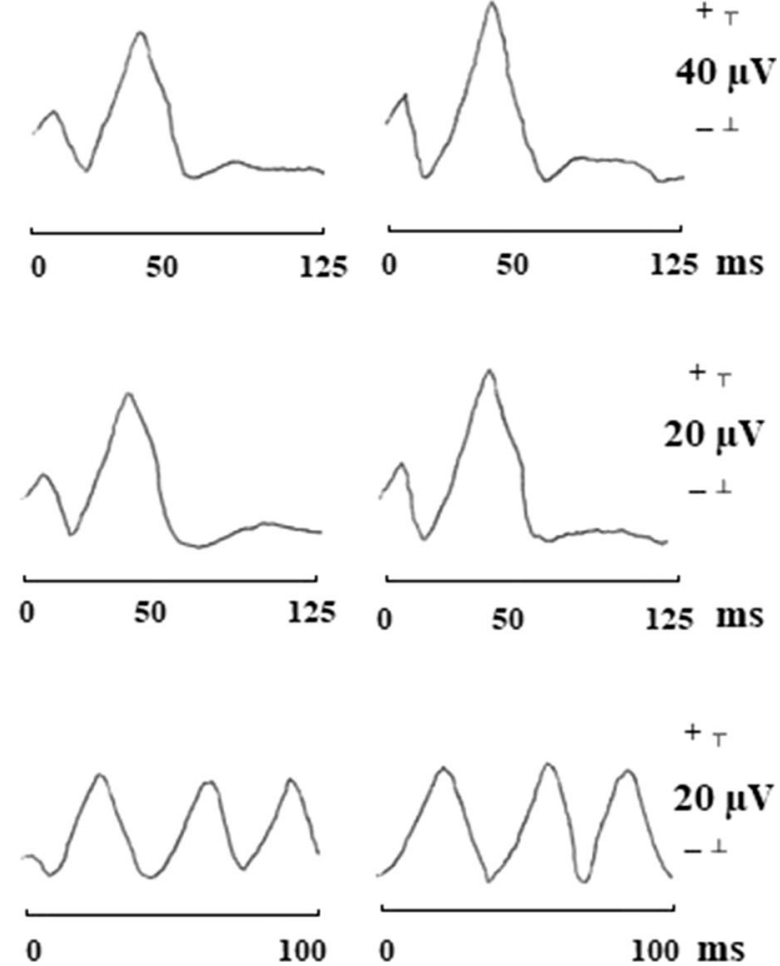

JS-NRD\#6 eye

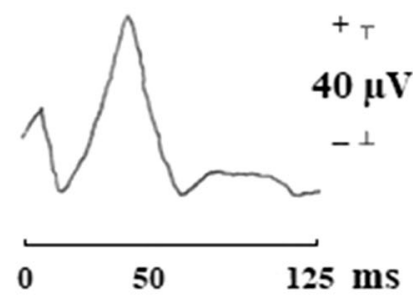

Fig. 1 Examples of dark-adapted electroretinogram (D-A ERG), light-adapted ERG (L-A ERG), and 30-Hz flicker ERG (F-ERG) responses assessed in one control subject (C\#12 eye), in one representative patient with Joubert syndrome with retinal dystrophy (JS-RD\#9 eye), and in one representative patient with Joubert syndrome without retinal dystrophy (JS-NRD\#6 eye). With respect to control

\section{RESULTS}

\section{Visual Acuity Data}

On average, a significant $(p<0.01)$ reduction in BCVA values was observed when data from the JS-RD group were compared to those of controls and of the JS-NDR group. In the JS-RD group, the greatest BCVA reduction was detected in patients with INPP5E, TMEM67, and C5orf42 mutations. In the JS-NDR group, no significant $(p>0.01)$ differences in BCVA values were found with respect to those of controls. eye, both JS-RD and JS-NRD eyes showed light-adapted ERG and dark-adapted ERG with a delay in a- and b-wave implicit times (dashed lines) and with a reduction in a- and b-wave peak-to-peak amplitudes (arrows) and a reduction of F-ERG peak-to-peak amplitudes (arrows). ms milliseconds, $\mu \mathrm{V}$ microvolt

\section{ERG Analysis}

In Fig. 1 are reported representative examples of dark-adapted ERG, light-adapted ERG, and flicker ERG responses assessed in one control subject (C\#12 eye), in one JS patient with retinal dystrophy (JS-RD\#9 eye) and in one patient without retinal dystrophy (JS-NRD\#6 eye).

On Table 2 are reported the correlation between the individual values of dark-adapted ERG, light-adapted ERG, and flicker ERG parameters and the corresponding values of BCVA observed in JS-RD and JS-NRD groups.

Table 3 presents the mean data of lightadapted ERG, dark-adapted ERG, and flicker 


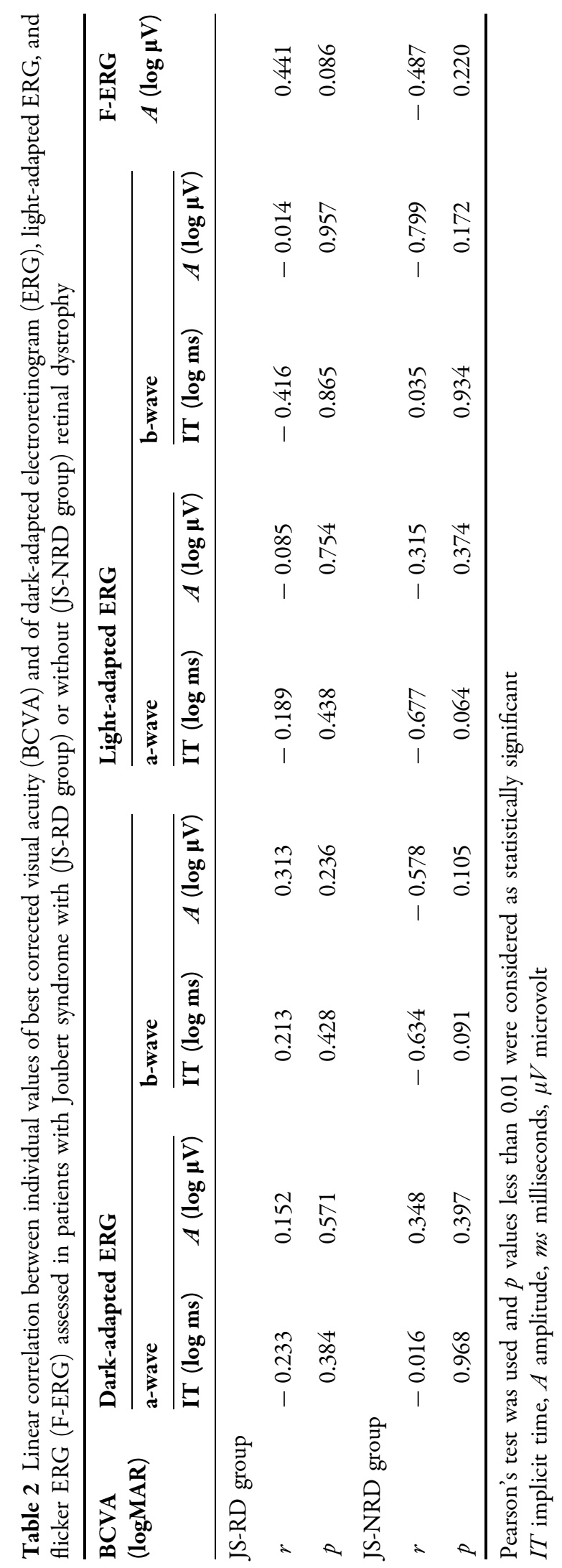


Table 3 Mean values of dark-adapted electroretinogram (ERG), light-adapted ERG, and 30-Hz flicker ERG (F-ERG) responses assessed in control subjects (C) and in patients with Joubert syndrome with (JS-RD group) or without (JS-NRD group) retinal dystrophy

\begin{tabular}{|c|c|c|c|c|c|c|c|c|c|}
\hline & \multicolumn{4}{|c|}{ Dark-adapted ERG } & \multicolumn{4}{|c|}{ Light-adapted ERG } & \multirow{3}{*}{$\begin{array}{l}\text { F-ERG } \\
A(\log \mu \mathrm{V})\end{array}$} \\
\hline & \multicolumn{2}{|l|}{ a-wave } & \multicolumn{2}{|l|}{ b-wave } & \multicolumn{2}{|l|}{$\overline{\text { a-wave }}$} & \multicolumn{2}{|l|}{ b-wave } & \\
\hline & $\begin{array}{l}\text { IT } \\
(\log \mathrm{ms})\end{array}$ & $A(\log \mu \mathrm{V})$ & $\begin{array}{l}\text { IT } \\
(\log \mathrm{ms})\end{array}$ & $A(\log \mu \mathrm{V})$ & $\begin{array}{l}\text { IT } \\
(\log \mathrm{ms})\end{array}$ & $A(\log \mu \mathrm{V})$ & $\begin{array}{l}\text { IT } \\
(\log \mathrm{ms})\end{array}$ & $A(\log \mu \mathrm{V})$ & \\
\hline \multicolumn{10}{|c|}{$\mathrm{C}(N=25)$} \\
\hline Mean & 13.271 & 46.125 & 28.875 & 71.542 & 11.042 & 12.933 & 28.813 & 29.355 & 34.063 \\
\hline SD & 0.766 & 2.597 & 2.849 & 4.688 & 1.444 & 2.083 & 1.131 & 3.852 & 2.288 \\
\hline \multicolumn{10}{|c|}{$\mathrm{JS}-\mathrm{RD}(N=16)$} \\
\hline Mean & 15.613 & 26.081 & 41.738 & 38.110 & 13.069 & 8.819 & 34.047 & 16.469 & 15.806 \\
\hline SD & 3.111 & 10.369 & 10.333 & 20.099 & 2.786 & 4.486 & 8.827 & 10.498 & 9.138 \\
\hline \multicolumn{10}{|c|}{$\mathrm{AV}$ vs $\mathrm{C}$} \\
\hline$f(1,40)$ & 13.101 & 86.142 & 35.053 & 64.561 & 9.392 & 16.332 & 8.692 & 31.444 & 92.022 \\
\hline$p$ & $<0.001$ & $<0.001$ & $<0.001$ & $<0.001$ & $<0.001$ & $<0.001$ & $<0.001$ & $<0.001$ & $<0.001$ \\
\hline \multicolumn{10}{|c|}{ JS-NRD $(N=8)$} \\
\hline Mean & 15.275 & 29.325 & 37.938 & 43.066 & 15.000 & 8.625 & 29.188 & 18.425 & 20.613 \\
\hline SD & 2.647 & 14.336 & 5.421 & 18.930 & 4.520 & 4.211 & 1.624 & 5.129 & 14.102 \\
\hline \multicolumn{10}{|c|}{$\mathrm{AV}$ vs $\mathrm{C}$} \\
\hline$f(1,32)$ & 11.951 & 33.133 & 45.644 & 50.182 & 15.252 & 15.283 & 0.543 & 41.543 & 22.392 \\
\hline$p$ & 0.002 & $<0.001$ & $<0.001$ & $<0.001$ & $<0.001$ & $<0.001$ & 0.469 & $<0.001$ & $<0.001$ \\
\hline \multicolumn{10}{|c|}{ AV vs JS-RD } \\
\hline$f(1,23)$ & 0.073 & 0.402 & 0.944 & 0.345 & 1.692 & 0.954 & 2.330 & 0.241 & 1.032 \\
\hline$p$ & 0.795 & 0.531 & 0.343 & 0.567 & 0.208 & 0.341 & 0.141 & 0.626 & 0.322 \\
\hline
\end{tabular}

$A N O V A$ statistical evaluation by one-way analysis of variance between groups. $p$ values less than 0.01 were considered as statistically significant (in bold)

$N$ number of eyes, $S D$ standard deviation, $I T$ implicit time, $A$ amplitude, $m s$ milliseconds, $\mu V$ microvolt

ERG parameters detected in controls and JS-RD and JS-NRD groups together with a relative statistical analysis between groups.

The ERG results are reported separately in the following subsections.

\section{Dark-Adapted ERG}

On average, in the JS-RD group a-wave and b-wave ITs and $A$ values were respectively significantly $(p<0.01)$ increased and reduced when compared to control ones.

In the JS-RD group, the greatest delay of $a$-wave and b-wave IT and the greatest reduction of a-wave and b-wave $A$ were observed in patients with INPP5E mutation.

Considering individual patients with JS-RD, the a-wave and b-wave ITs and $A$ values were not significantly $(p>0.01)$ correlated with the corresponding values of BCVA. 
On average, when compared to those of controls, in the JS-NRD group a significant $(p<0.01)$ increase in a-wave and b-wave IT values and a significant $(p<0.01)$ reduction in a-wave and b-wave $A$ values were found. JS-NRD and JS-RD groups showed no significant $(p>0.01)$ differences between the mean values of all dark-adapted ERG parameters. When considering the individual JS-NRD values, the greatest delay of a-wave IT, the greatest reduction in b-wave $A$, and the greatest reduction of a-wave $A$ were found in patients with CC2D2A mutation, whereas the greatest reduction of a-wave $A$ and delay of b-wave IT were detected in patients with C5orf42 mutation.

In JS-NRD, no significant $(p>0.01)$ linear correlation between the individual values of a-wave and b-wave ITs and $A$ values with the corresponding values of BCVA was observed.

\section{Light-Adapted ERG}

On average, in the JS-RD group a significant $(p<0.01)$ increase in a-wave and b-wave ITs and a significant $(p<0.01)$ reduction in a-wave and b-wave $A$ values were found with respect to control ones.

Considering the individual values detected in patients with JS-RD, the greatest increase of $a$-wave and b-wave IT and the greatest reduction of a-wave and b-wave $A$ values were found in patients with INPP5E mutation.

The increase of a-wave and b-wave ITs and the reduction of a-wave and b-wave $A$ values observed in individual patients with JS-RD were not significantly $(p>0.01)$ linearly correlated with the corresponding values of BCVA.

On average, in the JS-NRD group a significant $(p<0.01)$ increase in a-wave IT values and a significant $(p<0.01)$ reduction in a-wave and $\mathrm{b}$-wave $A$ values with respect to those of control ones were detected. No significant $(p>0.01)$ differences were observed for the b-wave IT values between JS-NRD and control groups. No significant $(p>0.01)$ differences of the mean values of all light-adapted ERG parameters were found between the JS-RD and JS-NRD groups.

In the JS-NRD group, when considering the individual values, the greatest delay of a-wave and $b$-wave IT in the greatest reduction of a-wave $A$ were found in patients with $C 5$ orf 42 mutation, whereas the greatest reduction of b-wave $A$ was observed in patients with $C C 2 D 2 A$ mutation. In the JS-NRD group, no significant $(p>0.01)$ linear correlations were observed between the individual values of a-wave and b-wave ITs and $A$ values and the corresponding values of BCVA.

\section{Flicker ERG}

On average, in the JS-RD group, significantly $(p<0.01)$ reduced amplitude values were found with respect to control ones. In this group, the greatest reduction of amplitude was observed in patients with INPP5 mutation. Considering individual patients with JS-RD, amplitudes were not significantly $(p>0.01)$ linearly correlated with the corresponding values of BCVA. On average, when compared to controls, in the JSNRD group a significant $(p<0.01)$ reduction in amplitude was found. JS-RD and JS-NRD groups showed no significant $(p>0.01)$ differences in amplitude values.

Considering individual JS-NRD values, the greatest reduction of amplitude was detected in patients with $C C 2 D 2 A$ mutation. In JS-NRD, no significant $(p>0.01)$ linear correlations were observed between the individual amplitude values and the corresponding values of BCVA.

\section{DISCUSSION}

The aim of our work was to evaluate the retinal function (by ERG recordings) in patients with JS with or without retinal dystrophy. As a consequence of the ERG findings, this technique could be suggested as an objective method for the evaluation of effectiveness of credible treatment in future clinical trials.

In our study, we performed three different types of ERG recordings that allowed us to evaluate selectively the function of different retinal elements. In particular, in accordance with ISCEV standards for clinical electroretinography, we recorded dark-adapted ERG that is a combined response arising from photoreceptors (a-wave) and bipolar cells (b-wave) of both the rod and cone systems (rod dominated), light-adapted ERG that reflects the bioelectrical activity of the cone (a-waves), and 
cone ON and OFF bipolar cells (b-wave), and light-adapted $30-\mathrm{Hz}$ flicker ERG that evaluates the cone-pathway-driven response [16].

In our patients with JS-RD we found significant abnormal dark- and light-adapted ERG and flicker ERG responses when compared to controls. Our findings are consistent with the very few articles present in the literature [7, 18, 19]. Our results suggest that in patients with JS-RD there is a dysfunction of both rod and cone systems and ON and OFF bipolar cells [16]. The observed dysfunction can be ascribed to both rod and cone disorganization of photoreceptor outer segments. This is suggested by the findings obtained in animal models with an electron microscopy evaluation [20, 21], and by morphological evidence in human studies performed by using optical coherence tomography assessment $[22,23]$.

In our cohort of patients with JS-RD, the greatest ERG abnormalities were detected in patients with INPP5E mutation and this may represent a novel finding since in other previous similar published works $[7,18,19]$ there is a lack of information about the relationship between the increase of a- and b-wave ITs and the reduction of a- and b-wave $A$ values and the specific detected mutation.

The genetic mutation can impact at different levels and times in retinal development and metabolism, resulting in a retinal functional and morphological involvement. All this can be explained by the data that in all JS genetic types, the mutation implies a Wnt or hedgehog (Shh) signal dysfunction and it is the role of Wht and Shh genes as regulator for normal laminar organization of the retinal elements is well known [24-27]. In addition, the lack or a dysfunction of Wnt may induce a retinal pigmented epithelium hypertrophy or hyperplasia (as observed in 8 of our 16 patients with JS-RD) and failure of ventral retinal development originating in a retinal coloboma (as observed in 4 of our 16 patients with JS-RD) [27].

In our JS-RD group, large BCVA reduction was detected in patients with INPP5E, TMEM67, and C5orf42 mutations and this is consistent with all data previously reported by Brooks et al. [19].
In the JS-RD group, no significant correlations were found between the reduction of BCVA and ERG abnormalities. This can because the data we obtained by performing different types of ERG recordings reflect the bioelectrical activity of different retinal elements of the whole retina with a negligible contribution of the macular region, which is strictly implicated in the visual acuity assessment. For this requirement, it would be useful to perform multifocal ERG recordings that, by applying the "ring analysis" [28-33], allow a functional evaluation of cones and bipolar cells located in the macular area [34]. Nevertheless, the multifocal ERG recordings require a particular ability to maintain a stable fixation for the entire duration of the examination and we believe that cannot be correctly performed in children with a low visual acuity such as our patients with JS-RD.

An interesting finding of our work is that significant abnormal ERG values (dark- and light-adapted and flicker responses) were also detectable in patients with JS without retinal dystrophy (JS-NRD group). Such as for other diseases (i.e., diabetes without retinopathy) [35], a retinal dysfunction, in the absence of retinal dystrophy at fundus examination, may also occur.

The observed ERG findings may suggest that all previously cited retinal development and metabolism changes [24-27] can also occur patients in the JS-NRD group, inducing a dysfunction of both rod and cone systems and ON and OFF bipolar cells.

Since in the JS-NRD group no significant changes in BCVA with respect to controls were found, it is likely that the macular area is spared from this involvement; however, to exclude this possibility a multifocal ERG assessment would be required. Also in this case, the same aforementioned limitation (ability to maintain a stable fixation) persists.

Our cohort of patients with JS-NRD comprised only eight children whereas there were 16 patients with JS-RD enrolled; this is in accordance with data reporting a less frequent absence of retinal dystrophy in this syndrome $[5,20]$. The greatest ERG abnormalities were found in patients with both CC2D2A and 
C5orf42 mutations, whereas in the JS-RD group it was in patients with INPP5E mutation. It is worth noting that none of our enrolled patients with JS-NRD had INPP5E mutation. Our ERG findings are similar to that reported in only one case by Brooks et al., although the mutation was not specified [19].

All ERG findings detected in our patients with JS were similar to those observed in patients with other retinal dystrophies (see Creel for a review [36]). Recently, to obtain an increase of retinal function, several clinical trials have been performed in patients with inherited retinal dystrophies by using different types of treatments. In many of these studies [37-44] the efficacy of the treatment was evaluated by measuring the changes of the ERG responses. Actually, no similar studies have been performed or are ongoing in patients with JS with retinal functional or morphological involvement.

\section{CONCLUSION}

Our results suggest that a dysfunction of photoreceptors and bipolar cells occurs in patients with JS with or without retinal dystrophy. The retinal impairment can be detected by ERG recordings, evaluating objectively the retinal function changes; this method, associated with psychophysical visual function measures (visual acuity and visual field), may be proposed as a tool able to evaluate the effectiveness of adequate treatment, targeted to improve the retinal impairment in patients with JS.

\section{ACKNOWLEDGEMENTS}

We thank the participants of the study. The contribution of the Bietti Foundation for this study was supported by the Italian Ministry of Health and Fondazione Roma.

Funding. No funding or sponsorship was received for this study or publication of this article.
Authorship. All named authors meet the International Committee of Medical Journal Editors (ICMJE) criteria for authorship for this article, take responsibility for the integrity of the work as a whole, and have given their approval for this version to be published.

Disclosures. Giulio Ruberto, Vincenzo Parisi, Chiara Bertone, Sabrina Signorini, Mauro Antonini, Enza Maria Valente, Federica Manzoni, Valentina Serpieri and Riccardo Fausto declare that they have no conflict of interest. Luciano Quaranta is a member of the journal's Editorial Board.

Compliance with Ethics Guidelines. All procedures performed in this study were in accordance with the ethical standards of the institutional and/or national research committee and with the 1964 Helsinki declaration and its later amendments or comparable ethical standards. The study was approved by the local institutional review board (Scientific Committee of Section of Ophthalmology, University of Pavia, IRCCS Fondazione Policlinico San Matteo, Pavia, Italy). Informed consent was obtained from all individual participants included in the study.

Data Availability. All authors had full access to all of the data in this study and take complete responsibility for the integrity of the data and accuracy of the data analysis. The datasets generated and/or analyzed during the current study are available from the corresponding author on reasonable request.

Open Access. This article is licensed under a Creative Commons Attribution-NonCommercial 4.0 International License, which permits any non-commercial use, sharing, adaptation, distribution and reproduction in any medium or format, as long as you give appropriate credit to the original author(s) and the source, provide a link to the Creative Commons licence, and indicate if changes were made. The images or other third party material in this article are included in the article's Creative Commons licence, unless indicated otherwise in a credit line to the material. If 
material is not included in the article's Creative Commons licence and your intended use is not permitted by statutory regulation or exceeds the permitted use, you will need to obtain permission directly from the copyright holder. To view a copy of this licence, visit http:// creativecommons.org/licenses/by-nc/4.0/.

\section{REFERENCES}

1. Joubert M, Eisenring JJ, Robb JP, Andermann F. Familial agenesis of the cerebellar vermis. A syndrome of episodic hyperpnea, abnormal eye movements, ataxia, and retardation. Neurology. 1969;19:813-25.

2. Poretti A, Boltshauser E, Valente EM. The molar tooth sign is pathognomonic for Joubert syndrome! Pediatr Neurol. 2014;50:e15-e1616.

3. Parisi MA. Clinical and molecular features of Joubert syndrome and related disorders. Am J Med Genet C Semin Med Genet. 2009;151C:326-40.

4. Stephen J, Vilboux T, Mian L, et al. Mutations in KIAA0753 cause Joubert syndrome associated with growth hormone deficiency. Hum Genet. 2017;136: 399-408.

5. Vilboux T, Doherty DA, Glass IA, et al. Molecular genetic findings and clinical correlations in 100 patients with Joubert syndrome and related disorders prospectively evaluated at a single center. Genet Med. 2017;19:875-82.

6. Mitchison MM, Valente EM. Motile and non-motile cilia in human pathology: from function to phenotypes. J Pathol. 2017;241:294-309.

7. Khan AO, Oystreck DT, Seidahmed MZ, et al. Ophthalmic features of Joubert syndrome. Ophthalmology. 2008;115:2286-9.

8. Dekaban AS. Hereditary syndrome of congenital retinal blindness (Leber), polycystic kidneys and maldevelopment of the brain. Am J Ophthalmol. 1969;68:1029-37.

9. Dekaban AS. Familial occurrence of congenital retinal blindness and developmental renal lesions. J Genet Hum. 1969;17:289-96.

10. Aicardi J, Castello-Branco ME, Roy C. Joubert's syndrome. Apropos of 5 cases. Arch Fr Pediatr. 1983;40:625-9.
11. Tusa RJ, Hove MT. Ocular and oculomotor signs in Joubert syndrome. J Child Neurol. 1999;14:621-7.

12. Moore AT, Taylor DS. A syndrome of congenital retinal dystrophy and saccade palsy-a subset of Leber's amaurosis. Br J Ophthalmol. 1984;68: 421-31.

13. Laverda AM, Saia OS, Drigo P, Danieli E, Clementi $\mathrm{M}$, Tenconi R. Chorioretinal coloboma and Joubert syndrome: a nonrandom association. J Pediatr. 1984;105:282-4.

14. Lindhout D, Barth PG, Valk J, Boen-Tan TN. The Joubert syndrome associated with bilateral chorioretinal coloboma. Eur J Pediatr. 1980;134:173-6.

15. Appleton RE, Chitayat D, Jan JE, Kennedy R, Hall JG. Joubert's syndrome associated with congenital ocular fibrosis and histidinemia. Arch Neurol. 1989;46:579-82.

16. McCulloch DL, Marmor MF, Brigell MG, et al. ISCEV standard for full-field clinical electroretinography (2015 update). Doc Ophthalmol. 2015;130:1-12.

17. Roosing S, Romani M, Isrie $\mathrm{M}$, et al. Mutations in CEP120 cause Joubert syndrome as well as complex ciliopathy phenotypes. J Med Genet. 2016;53: 608-15.

18. Abdelhamed ZA, Wheway G, Szymanska K, et al. Variable expressivity of ciliopathy neurological phenotypes that encompass Meckel-Gruber syndrome and Joubert syndrome is caused by complex de-regulated ciliogenesis, Shh and Wnt signalling defects. Hum Mol Genet. 2013;22:1358-72.

19. Brooks BP, Zein WM, Thompson AH, et al. Joubert syndrome: ophthalmological findings in correlation with genotype and hepatorenal disease in 99 patients prospectively evaluated at a single center. Ophthalmology. 2018;125:1937-52.

20. Bachmann-Gagescu R, Dempsey JC, Phelps IG, et al. Joubert syndrome: a model for untangling recessive disorders with extreme genetic heterogeneity. J Med Genet. 2015;52:514-22.

21. Westfall JE, Hoyt C, Liu Q, et al. Retinal degeneration and failure of photoreceptor outer segment formation in mice with targeted deletion of the Joubert syndrome gene, Ahi1. J Neurosci. 2010;30: 8759-68.

22. Baba S, Takeshita E, Yamazaki H, Tarashima M, Sasaki M. Disruption of the photoreceptor inner segment-outer segment junction in a 6-year-old girl with Joubert syndrome. Neuroophthalmology. 2016;41:19-23. 
23. Bonilha VL, Rayborn ME, Bell BA, et al. Histopathology of the retina from a three year-old suspected to have Joubert syndrome. Austin J Clin Ophthalmol. 2015;2:1057.

24. Valente EM, Logan CL, Mougou-Zerelli S, et al. Mutations in TMEM216 perturb ciliogenesis and cause Joubert Meckel and related syndromes. Nat Genet. 2010;42:619-25.

25. Garcia-Gonzalo FR, Corbit KC, Sirerol-Piquer MS, et al. A transition zone complex regulates mammalian ciliogenesis and ciliary membrane composition. Nat Genet. 2011;43:776-84.

26. Wang YP, Dakubo G, Howley P, et al. Development of normal retinal organization depends on sonic hedgehogh signaling from ganglion cells. Nat Neurosci. 2002;5:831-2.

27. Lad EM, Cheshier SH, Kalani MYS. Wnt-signaling in retinal development and disease. Stem Cells Dev. 2009;18:7-16.

28. Parisi V, Ziccardi L, Centofanti M, et al. Macular function in eyes with open-angle glaucoma evaluated by multifocal electroretinogram. Invest Ophthalmol Vis Sci. 2012;53:6973-80.

29. Ziccardi L, Parisi V, Picconi F, et al. Early and localized retinal dysfunction in patients with type 1 diabetes mellitus studied by multifocal electroretinogram. Acta Diabetol. 2018;55:1191-200.

30. Cascavilla ML, Parisi V, Triolo G, et al. Retinal dysfunction characterizes subtypes of dominant optic atrophy. Acta Ophthalmol. 2018;96: e156-e163163.

31. Parisi V, Ziccardi L, Stifano G, Montrone L, Gallinaro $G$, Falsini B. Impact of regional retinal responses on cortical visually evoked responses: multifocal ERGs and VEPs in the retinitis pigmentosa model. Clin Neurophysiol. 2010;121:380-5.

32. Ruberto G, Parisi V, Vandelli G, et al. Surgery for idiopathic epimacular membrane: morpho-functional outcomes based on the preoperative macular integrity of the photoreceptoral junction. A prospective pilot study. Adv Ther. 2020;37:566-77.

33. Parisi V, Ziccardi L, Costanzo E, et al. Macular functional and morphological changes in intermediate age-related maculopathy. Invest Ophthalmol Vis Sci. 2020;61:11.
34. Hood DC. Assessing retinal function with the multifocal technique. Prog Retin Eye Res. 2000;19: 607-46.

35. Parisi V, Uccioli L. Visual electrophysiological responses in persons with type 1 diabetes. Diabetes Metab Res Rev. 2001;17:12-8.

36. Creel DJ. Electroretinograms. Handb Clin Neurol. 2019;160:481-93.

37. Oner A, Gonen ZB, Sinim N, Cetin M, Ozkul Y. Subretinal adipose tissue-derived mesenchymal stem cell implantation in advanced stage retinitis pigmentosa: a phase I clinical safety study. Stem Cell Res Ther. 2016;7:178.

38. Hoffman DR, Hughbanks-Wheaton DK, et al. Fouryear placebo-controlled trial of docosahexaenoic acid in X-linked retinitis pigmentosa (DHAX trial): a randomized clinical trial. JAMA Ophthalmol. 2014;132:866-73.

39. Rayapudi S, Schwartz SG, Wang X, Chavis P. Vitamin A and fish oils for retinitis pigmentosa. Cochrane Database Syst Rev. 2013;12:CD008428.

40. Hoffman DR, Locke KG, Wheaton DH, Fish GE, Spencer R, Birch DG. A randomized, placebo-controlled clinical trial of docosahexaenoic acid supplementation for X-linked retinitis pigmentosa. Am J Ophthalmol. 2004;137:704-18.

41. Hoffman DR, Hughbanks-Wheaton DK, Spencer R, et al. Docosahexaenoic acid slows visual field progression in X-linked retinitis pigmentosa: ancillary outcomes of the DHAX trial. Invest Ophthalmol Vis Sci. 2015;56:6646-53.

42. Humayun MS, de Juan E Jr, del Cerro M, et al. Human neural retinal transplantation. Invest Ophthalmol Vis Sci. 2000;41:3100-6.

43. Petersen-Jones SM, Occelli LM, Biel M, Michalakis S. Advancing gene therapy for PDE6A retinitis pigmentosa. Adv Exp Med Biol. 2019;1185:103-7.

44. Talcott KE, Ratnam K, Sundquist SM, et al. Longitudinal study of cone photoreceptors during retinal degeneration and in response to ciliary neurotrophic factor treatment. Invest Ophthalmol Vis Sci. 2011;52:2219-26. 